Article

\title{
An Ethnobotanical Survey of Medicinal Plants Used in Papantla, Veracruz, Mexico
}

\author{
Eduardo Alberto Lara Reimers ${ }^{1}$, Eloy Fernández C. ${ }^{1, * \mathbb{D}}$, David J. Lara Reimers ${ }^{2}$, \\ Petra Chaloupkova ${ }^{3}$, Juan Manuel Zepeda del Valle ${ }^{4}$, Luigi Milella ${ }^{5,6, *(1)}$ and Daniela Russo 5,6 \\ 1 Department of Crop Sciences and Agroforestry, Faculty of Tropical AgriSciences, Czech University of Life \\ Sciences Prague, Praha 6-Suchdol, Kamýcká 129, 16500 Prague, Czech Republic \\ 2 Department of Forestry Engineering, Division of Forest Sciences, Autonomy University of Chapingo, \\ Km 38.5 roadway, México-Texcoco, Chapingo, CP 56230, Mexico \\ 3 Department of Economics and Development, Faculty of Tropical AgriSciences, Czech University of Life \\ Sciences Prague, Praha 6-Suchdol, Kamýcká 129, 16500 Prague, Czech Republic \\ 4 Department of Regional Rural Development, Autonomy University of Chapingo Zacatecas, \\ Zacatecas 98085, Mexico \\ 5 Department of Science, Basilicata University, V.le Ateneo Lucano 10, 85100 Potenza, Italy \\ 6 BioActiPlant s.r.l., Academic spinoff, Basilicata University, V.le Ateneo Lucano 10, 85100 Potenza, Italy \\ * Correspondence: eloy@ftz.czu.cz (E.F.C.); luigi.milella@unibas.it (L.M.)
}

Received: 21 June 2019; Accepted: 22 July 2019; Published: 24 July 2019

\begin{abstract}
An ethnobotanical study was performed to collect information on the use of medicinal plants in Papantla, Veracruz, Mexico. The area has a high number of endemic species, and the social importance of the medicinal plants in the community is essential for public health and the conservation of traditional knowledge. This study identified the medicinal plants currently used, registered traditional knowledge, and documented the patterns of ailments treated in the indigenous communities of Totonacas. A total of 101 medicinal plants belonging to 51 families were described by 85 local informants. Asteraceae was the family with the highest number of plant species identified by these informants. Plant parts are used to treat several ailments, including venomous bites, gastro-intestinal disorders, infectious diseases and other disorders. Informants reported that the most common plant part used was the leaf tissue $(55 \%)$, and they also took the herbal remedies orally $(72 \%)$, and decoctions ( $38 \%$ ) as well as infusions ( $29 \%)$ were the forms used to prepare these natural remedies. This study provides documentation of medicinal plants used in the Veracruz area of Mexico. Mexican people are still dependent upon medicinal plants, and in order to avoid their loss, certain measures of conservation for medicinal plants are needed.
\end{abstract}

Keywords: ethnobotany; medicinal plants; fidelity level; informant's consensus factor; use-reports

\section{Introduction}

Traditional medicine is considered the first health care resource to treat ailments in several countries [1-3], and about $80 \%$ of people in the world depend upon traditional medicine, according to the World Health Organization (WHO) [4].

Traditional medicine studies include ethnomedicine, which involves the practices most used by people that live in rural areas and indigenous communities, and this ethnomedicine is affected by factors such as age, gender, economic activity, socio-economic level, migration, access to new health care systems, new herbal products and urbanisation [5-7]. The diversity of medicinal plants is very high in Mexico; the country contains a wide variety of plants $(30,000)$ which have not been explored in their totality [8], and a significant number are endemic (uniquely native) species [9]. 
In Mexico, there are more than 50 indigenous languages spoken, and this country is very well-known for its biological diversity [10]. At the national level, the majority of Mexican indigenous populations live in rural areas (61.1\% in communities with less than 2500 inhabitants).

The gathering and use of local resources are still important aspects of the phytotherapeutic traditions in many regions of Mexico. Plants are also used for ornamental, nutritional (food and fodder), pharmaceutical, aromatic, religious or construction purposes [9]. Aspects such as the richness and diversity of cultures will increase the relative importance and roles (uses) that each plant could have in respective communities. Mexican medicinal plants play an important role in public health among the local communities. In fact, traditional medicine is the first route to treat ailments, because many drugs are expensive, or are not always available locally [11].

Mexico has 31 states and a federal district, and Veracruz is one of the richest with regard to its biological and cultural diversity [12]. The medicinal flora of Veracruz has been used as remedies to treat several diseases, including those of the digestive system, skin and reproductive system, or for religious-cultural practices (often referred as "limpias") [13]. In the Veracruz area, there are a number of indigenous groups who are keen consumers and practitioners of traditional medicine, such as the Totonacs, Tepehuas, Nahuas, Otomies, Popurcas, Zocos and the Popoluca Zaco, among others [14]. Previous studies carried out in the State of Veracruz registered more than 600 different taxa used for therapeutic purposes by the Popoluca (southern Veracruz) [15]. This supports the need to keep studying the areas previously studied in order to quantify and record these potential plants without antecedents. The wild flora in the state still play an important role, with different benefits for the social and ecological systems [16]. The Totonacs have a history of traditional medicine uses, and they are well-known for preserving a wide variety of plants. In the study area, as in other regions and countries, the recording of traditional knowledge is not widely promoted or supported, and the preservation of plant use knowledge is still carried out in oral form, and transmitted generation-by-generation [17]. Due to this fact, the aim of our work was to discover and document the traditional knowledge of medicinal plants in the 16 communities of Papantla, Veracruz, Mexico. A quantitative ethnobotanical approach was performed to analyse the medicinal use of plants and to select the important species in Papantla traditional medicine.

\section{Results and Discussion}

\subsection{Characteristics of the Informants}

There is a high prevalence in the use of medicinal plants and traditional knowledge in the Veracruz area. Table 1 provides the socio-demographic information such as residence, gender, age, occupation and annual expenditure in plants of the informants. Of the 85 indigenous people who answered the form, men represented the highest number (53\%), and people from 70 to 85 years appeared to have a more extensive knowledge of these plants. In general, the gender was not significantly correlated to age and plant knowledge $[18,19]$. Even though all of the respondents generally used traditional plants, it has been common that, in many parts of the world, the women always demonstrate a more extensive knowledge in the use of plants [20]. This could be due to their roles in the family.

Previous studies have shown that women usually have more knowledge about medicinal plants, and men know more about timber and handcraft species. This happens due to the sexual division of labour, and external factors resulting from the male migration of the young people inside the communities $[18,21]$. In our study, women on average cite two more plants and spend more money $(17$ plants, expenditure: \$200) than their male counterparts (15 plants, expenditure: \$176). Homemakers (\$210 annually) generally spend more money for plants compared to those in other occupations; the lowest expenditure was observed for the sellers. The expenditure for plants during the year is equivalent to two days of work for the farmers. Case, et al. [22] mentions that local knowledge in plants increases with increasing geographical isolation; the people intensively use more plant species 
due to the scarcity of medical centres, specialists or medicines. Nevertheless, other studies have related family income (economic status) as the main factor in the number and the uses of known plants [23].

Table 1. Socio-demographic characteristics of the 85 informants.

\begin{tabular}{ccccc}
\hline Background Characteristic & No & \% & $\begin{array}{c}\text { Annual Expenditure } \\
\text { (Mexican Pesos, \$) }\end{array}$ \\
\hline Residence & Rural & 26 & 31 & 160 \\
Gender & Urban & 59 & 69 & 200 \\
& Female & 40 & 47 & 174 \\
Age & Male & 45 & 53 & 200 \\
& $>20$ & 2 & 2 & 200 \\
& $21-30$ & 13 & 15 & 233 \\
& $31-40$ & 12 & 14 & 183 \\
& $41-50$ & 16 & 19 & 173 \\
& $51-60$ & 20 & 24 & 210 \\
& $61-70$ & 14 & 17 & 120 \\
& $71-85$ & 8 & 9 & 225 \\
& Farmer & 9 & 11 & 114 \\
& Housewife & 21 & 25 & 210 \\
& Seller & 4 & 5 & 50 \\
& Teacher & 2 & 2 & 200 \\
& Worker & 40 & 47 & 181 \\
& Other & 9 & 10 & 222 \\
\hline
\end{tabular}

The keepers of the traditional knowledge were found to be the teachers (average of 22 plants), followed by farmers (18 plants), and housewives (15 plants). Academic staff (teachers) showed more knowledge of plants because they received children from different communities, and the social engagement in those schools promoted and applied different strategies to keep their costumes, languages and traditions alive inside the classroom.

In Mexico, the preservation of plant use knowledge is still carried out in oral form and transmitted generation by generation. In fact, informants declare that the origin of the traditional knowledge was given by the family (87\%) and the specialist "shamans" (13\%). More than half of the informants (58\%) claimed to perceive a decrease (medium and high loss) in the traditional knowledge during their youth; the rest $(42 \%)$ did not perceive any alarming decrease.

Seventy-eight of the informants (92\%) were actively using medicinal plants in their daily lives, but 70 of these $(82 \%)$ recur to the plants in the first instance to treat their ailments. Nevertheless, there were just $16 \%$ (14 people) going to specialists. The informants obtained the plants mostly from wild gathering (37\%), the market (35\%) and familiar gardens $(28 \%)$.

Some informants $(29 \%)$ expressed that they do not have any expenditure in buying plants, since they know the surrounding areas where they can find them. The informants (38\%) positively emphasised the usage of medicinal plants as part of their cultural uses (38\%), and they consider them as an effective and cheap resource (35\%) since their childhood to treat human disorders.

\subsection{Mode of Preparation and Administration of Different Plant Parts}

Informants recognised 101 ethnobotanical plants belonging to 51 families distributed in 95 genera (Table 2), which were commonly used by most the of indigenous people for the treatments of 77 ailments. The most represented families were Asteraceae and Rutaceae, with eight plant species for each one, followed by Fabaceae (six species), Myrtaceae, Malvaceae and Apocynaceae (four species), and then Euphorbiaceae, Lamiaceae, Meliaceae and Poaceae with three species. Other families had two and one species each reported. Plant species of the Asteraceae family were the most used in Populoca, Veracruz [24], and not only in Papantla. This could be due to the abundance and wide variety of Asteraceae species in ecosystems in the northern and rainforest areas of Veracruz [16,18] and to their 
relative cultural importance. The plant list reported that a total of $61 \%$ of the species are native, $37 \%$ are exotic, and only $2 \%$ are endemic. The indigenous people keep using the local plants due to the great knowledge of the properties of these plants. The plant parts are usually consumed fresh $(83 \%)$, and leaves $(55 \%)$ represented the most common plant parts used by the informants to prepare their medicinal remedies. Leaves were followed by roots, bark, fruits, stems, whole plants, seeds, latex and flowers (Figure 1).

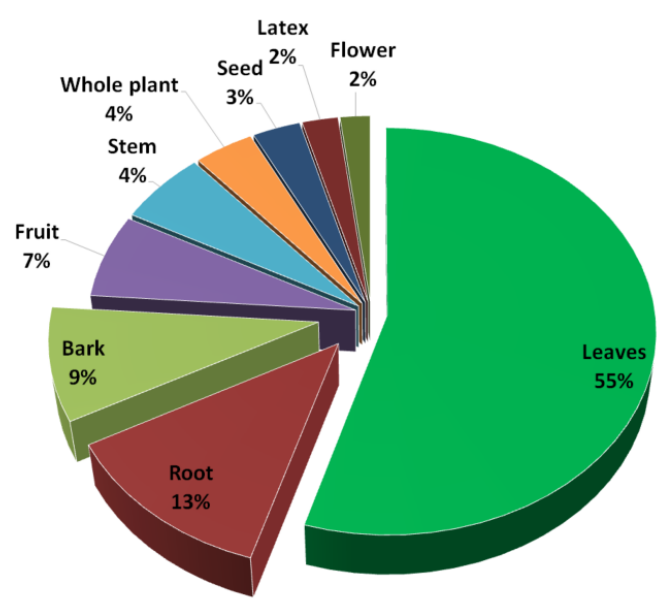

Figure 1. Plant parts used to prepare medicinal remedies.

The main reason for the use of leaves was the ease of collecting them. Herbal medicines are either based on single species or mixed with other plant species. Nowadays, the use of the multi-treatment (contemporary and conventional medicine) becomes a usual method for people to save money, obtain more results, and reduce the allopathic effects from the modern medicaments [25]. The mixture of two or more plants is seen in this study; and it is known that the use of more than two herbs could contain a range of different active compounds and can modify its effect, enhancing or reducing the healing effect. If we considered each mixture as one single remedy [26], the list of natural remedies could be multiplied. Nonetheless, the toxic effects should be studied in depth. Many of the plants were used in minimal concentrations by the locals, but they are still unknown and unregistered. It is remarkable that the use of exotic plants has not been well investigated against normal symptoms.

Two main routes of the administration of herbal remedies are reported: Oral (72\%) and topical $(28 \%)$ administration. Herbal remedies were prepared by using ten different methods. The main forms used were decoctions (38\%) and infusions (29\%), but raw plant material $(11 \%)$ was eaten fresh in order to combat gastrointestinal disorders, for blood circulation and against local pain (tooth pain). Raw materials of leaves, whole plants or stems were used for rituals, such as bad wind and evil eye. Bath and cataplasm (7\%) were applied to treat skin disorders (wounds) and to treat infective diseases such as chicken pox, smallpox and measles. Liquefied, crushed and smashed (5\%) remedies were used fresh and extracted from different plant parts for the treatment of diabetes and kidney problems. Squeezed $(4 \%)$, tinctured $(3 \%)$ and burned $(2 \%)$ remedies were mainly used to treat rheumatism, pain in the ears and wounds. The leaves of H. patens were used as therapy, where the leaves were burned and applied over the chest to promote breastfeeding in women. Bark, fruits and seeds were also fermented ( $2 \%$ ) to prepare alcoholic drinks (Figure 2). 
Table 2. Traditional use of medicinal plants among herbal practitioners in the study area (Totonac indigenous group).

\begin{tabular}{|c|c|c|c|c|c|c|c|c|c|c|c|}
\hline Family & Scientific Name & $\begin{array}{c}\text { Voucher } \\
\text { Specimen }\end{array}$ & $\begin{array}{l}\text { Common } \\
\text { Name }\end{array}$ & Origin & $\begin{array}{l}\text { Plant Part } \\
\text { Used }\end{array}$ & Ailments & Category & $\begin{array}{l}\text { Preparation } \\
\text { Mode }\end{array}$ & $\begin{array}{c}\text { Detailed } \\
\text { Administration }\end{array}$ & UR & FL (\%) \\
\hline \multirow[t]{2}{*}{ Amaranthaceae } & Beta vulgaris $\mathrm{L}$. & PA-01 & betabel & Ex & root & intestinal worms & G & fresh & raw & 1 & 100.0 \\
\hline & & & & & & stomach ache & G & fresh & infusion & 1 & \\
\hline \multirow[t]{3}{*}{ Amaryllidaceae } & Allium cepa $\mathrm{L}$. & PA-02 & cebolla morada & Ex & root & erection & $\mathrm{D}$ & fresh & crushed & 1 & 66.7 \\
\hline & & & & & & kidney problem & $\mathrm{C}$ & fresh & crushed & 1 & \\
\hline & & & & & & veterinary fever in chicken & $\mathbf{R}$ & fresh & smashed & 4 & \\
\hline \multirow[t]{6}{*}{ Amaryllidaceae } & Allium sativum $\mathrm{L}$. & PA-03 & ajo & Ex & root & blood circulation & B & fresh & infusion & 3 & 26.3 \\
\hline & & & & & & cold & A & fresh & infusion & 2 & \\
\hline & & & & & & grains in the skin & $\mathrm{O}$ & fresh & bath & 2 & \\
\hline & & & ajo con alcohol & & root & liver problems & $\mathrm{N}$ & fresh & microdosis & 1 & \\
\hline & & & & & & rheumatism & E & fresh & tincture & 5 & \\
\hline & & & & & & snake bites & $\mathrm{M}$ & fresh & tincture & 1 & \\
\hline \multirow[t]{2}{*}{ Amaryllidaceae } & Allium sativum $\mathrm{L}$. & & ajo con (with) & & leaves & stomach ache & G & fresh & infusion & 3 & \\
\hline & & & & & & tooth pain & $\mathrm{L}$ & fresh & raw & 2 & \\
\hline \multirow[t]{3}{*}{ Anacardiaceae } & Spondias mombin $\mathrm{L}$. & PA-04 & $\begin{array}{l}\text { caña con jobo, } \\
\text { durazno y piña }\end{array}$ & $\mathrm{N}$ & bark & alcoholic drink & $\mathrm{R}$ & dry & fermented & 1 & 66.7 \\
\hline & & & & & & flu & A & dry & decocted & 6 & \\
\hline & & & & & & tooth pain & $\mathrm{L}$ & dry & decocted & 2 & \\
\hline \multirow[t]{5}{*}{ Annonaceae } & Annona glabra $\mathrm{L}$. & PA-05 & anona & $\mathrm{N}$ & leaves & diarrhoea & G & fresh & infusion & 7 & 57.9 \\
\hline & & & & & fruit & drink & $\mathrm{s}$ & fresh & & 3 & \\
\hline & & & & & leaves & stomach ache & G & fresh & infusion & 4 & \\
\hline & & & & & fruit & to have children & $\mathrm{D}$ & dry & squeezed & 3 & \\
\hline & & & & & leaves & to have children & $\mathrm{D}$ & fresh & infusion & 2 & \\
\hline Annonaceae & Mangifera indica $\mathrm{L}$ & PA-06 & mango & Ex & seed & diarrhoea & G & fresh & decocted & 4 & 100.0 \\
\hline Apiaceae & Arracacia atropurpúrea & PA-07 & comino & $\mathrm{N}$ & leaves & diarrhoea & G & dry & decocted & 6 & 100.0 \\
\hline \multirow{3}{*}{ Apiáceas } & Annona muricata & PA-08 & guanabana & $\mathrm{N}$ & leaves & cancer & I & fresh & infusion & 7 & 34.4 \\
\hline & & & & & leaves & diabetes & $\mathrm{H}$ & fresh & infusion & 6 & \\
\hline & & & & & leaves & high pressure & B & fresh & infusion & 11 & \\
\hline Apiáceas & Apium graveolens $\mathrm{L}$. & & apio & Ex & stem & cholesterol & B & fresh & liquefied & 8 & 100.0 \\
\hline Apocynaceae & $\begin{array}{l}\text { Pentalinon andrieuxul } \\
\text { (Müll.Arg.) B.F.Hansen \& } \\
\text { Wunderlin }\end{array}$ & PA-09 & $\begin{array}{c}\text { guaco } \\
\text { enredadera }\end{array}$ & $\mathrm{N}$ & root & snake bites & M & dry & tincture & 13 & 100.0 \\
\hline Apocynaceae & Rauvolfia tetraphylla L. & PA-10 & cancerina & $\mathrm{N}$ & leaves & cancer & I & fresh & infusion & 11 & 100.0 \\
\hline \multirow[t]{4}{*}{ Arecaceae } & Cocos nucifera $\mathrm{L}$. & PA-11 & coco and caña & Ex & leaves & blood circulation & B & fresh & raw & 3 & 68.8 \\
\hline & & & & & & chinkunguya & $\mathbf{P}$ & fresh & raw & 5 & \\
\hline & & & & & & dengue & $\mathbf{P}$ & fresh & raw & 6 & \\
\hline & & & & & bark & stop bleeding in the parthum & $\mathrm{D}$ & fresh & decocted & 2 & \\
\hline
\end{tabular}


Table 2. Cont.

\begin{tabular}{|c|c|c|c|c|c|c|c|c|c|c|c|}
\hline Family & Scientific Name & $\begin{array}{c}\text { Voucher } \\
\text { Specimen }\end{array}$ & $\begin{array}{l}\text { Common } \\
\text { Name }\end{array}$ & Origin & $\begin{array}{l}\text { Plant Part } \\
\text { Used }\end{array}$ & Ailments & Category & $\begin{array}{l}\text { Preparation } \\
\text { Mode }\end{array}$ & $\begin{array}{c}\text { Detailed } \\
\text { Administration } \\
\end{array}$ & UR & FL (\%) \\
\hline \multirow[t]{2}{*}{ Arecáceas } & \multirow[t]{2}{*}{$\begin{array}{l}\text { Acrocomia aculeata (Jacq.) } \\
\text { Lodd. ex Mart. }\end{array}$} & \multirow[t]{2}{*}{ PA-12 } & \multirow[t]{2}{*}{$\begin{array}{l}\text { coyol redondo } \\
\text { palma }\end{array}$} & \multirow[t]{2}{*}{$\mathrm{N}$} & root & diabetes & $\mathbf{H}$ & dry & infusion & 3 & 50.0 \\
\hline & & & & & bark & eyes problem & $\mathbf{Q}$ & dry & decocted & 3 & 50.0 \\
\hline \multirow[t]{3}{*}{ Asclepiadaceae } & \multirow[t]{3}{*}{ Asclepias curassavica $\mathrm{L}$. } & \multirow[t]{3}{*}{ PA-13 } & \multirow[t]{3}{*}{ hierva del sapo } & \multirow[t]{3}{*}{$\mathrm{N}$} & leaves & cancer & $\tilde{I}$ & fresh & decocted & 16 & $59 ., 3$ \\
\hline & & & & & leaves & diabetes & $\mathrm{H}$ & dry & infusion & 5 & \\
\hline & & & & & leaves & kidney problem & C & fresh & decocted & 6 & \\
\hline Asteraceae & Artemisia ludoviciana Nutt. & PA-14 & estafiate & $\mathrm{N}$ & stem & cholesterol & B & fresh & infusion & 12 & 100.0 \\
\hline \multirow[t]{2}{*}{ Asteraceae } & \multirow[t]{2}{*}{$\begin{array}{c}\text { Calea ternifolia Oliv. ex } \\
\text { Thurn }\end{array}$} & \multirow[t]{2}{*}{ PA-15 } & \multirow[t]{2}{*}{ zacate chichi } & \multirow[t]{2}{*}{$\mathrm{N}$} & \multirow[t]{2}{*}{$\begin{array}{l}\text { leaves, } \\
\text { stem, } \\
\text { flower }\end{array}$} & bile & G & fresh & infusion & 5 & 70.6 \\
\hline & & & & & & diabetes & $\mathbf{H}$ & fresh & infusion & 12 & \\
\hline Asteraceae & $\begin{array}{c}\text { Cyclolepis genistoides } \\
\text { D.Don }\end{array}$ & PA-16 & palo azul & Ex & bark & kidney problem & C & dry & decocted & 4 & 100.0 \\
\hline Asteraceae & $\begin{array}{l}\text { Gnaphalium viscosum } \\
\text { Kunth }\end{array}$ & PA-17 & gordolobo & $\mathrm{N}$ & $\begin{array}{l}\text { whole } \\
\text { plant }\end{array}$ & cough & A & fresh & infusion & 2 & 100.0 \\
\hline \multirow[t]{3}{*}{ Asteraceae } & \multirow[t]{3}{*}{ Matricaria chamomilla $\mathrm{L}$. } & \multirow[t]{3}{*}{ PA-18 } & \multirow[t]{3}{*}{ manzanilla } & \multirow[t]{3}{*}{ Ex } & \multirow{3}{*}{$\begin{array}{c}\text { whole } \\
\text { plant } \\
\text { leaves } \\
\text { whole } \\
\text { plant }\end{array}$} & colic pain & D & fresh & decocted & 16 & 47.1 \\
\hline & & & & & & eyes problem & $\mathrm{Q}$ & fresh & bath & 4 & \\
\hline & & & & & & stomach ache & G & fresh & decocted & 14 & \\
\hline \multirow[t]{4}{*}{ Asteraceae } & \multirow[t]{4}{*}{$\begin{array}{c}\text { Parthenium hysterophorus } \\
\text { L. }\end{array}$} & \multirow[t]{4}{*}{ PA-19 } & \multirow[t]{4}{*}{$\begin{array}{l}\text { chuchullate o } \\
\text { tres hojitas }\end{array}$} & \multirow[t]{4}{*}{$\mathrm{N}$} & stem & anaemia & B & fresh & decocted & 1 & 53.8 \\
\hline & & & & & leaves & diabetes & H & fresh & infusion & 7 & \\
\hline & & & & & leaves & wounds & $\mathrm{O}$ & fresh & bath & 2 & \\
\hline & & & & & & wounds & $\mathrm{O}$ & fresh & decocted & 3 & \\
\hline Asteraceae & Tagetes erecta $\mathrm{L}$. & PA-20 & flor de muerto & $\mathrm{N}$ & root & stomach ache & G & dry & decocted & 6 & 100.0 \\
\hline \multirow[t]{4}{*}{ Asteraceae } & Verbesina persicifolia D.C & PA-21 & huichin & $\mathrm{N}$ & leaves & diabetes & $\mathbf{H}$ & fresh & infusion & 9 & 39.1 \\
\hline & & & & & leaves & gastritis & G & fresh & decocted & 1 & \\
\hline & & & & & root & high pressure & B & fresh & decocted & 9 & 39.1 \\
\hline & & & & & root & inflammation & E & fresh & bath & 4 & \\
\hline Bignoniaceae & $\begin{array}{l}\text { Parmentiera aculeata } \\
\text { (Kunth) Seem. }\end{array}$ & PA-22 & chote, chiote & $\mathrm{N}$ & flower & veterinary uses & $\mathrm{R}$ & fresh & decocted & 7 & 50.0 \\
\hline & & & & & & kidney problem & C & fresh & decocted & 7 & \\
\hline Bignoniaceae & $\begin{array}{c}\text { Tecoma stans (L.) Juss. ex } \\
\text { Kunth }\end{array}$ & PA-23 & $\begin{array}{l}\text { tronadora (hoja } \\
\text { de san pedro) }\end{array}$ & $\mathrm{N}$ & leaves & infection in skin & $\mathrm{O}$ & fresh & burned & 2 & 100.0 \\
\hline Brassicaceae & Nasturtium officinale R. Br. & PA-24 & berros & $\mathrm{N}$ & leaves & anaemia & B & fresh & bath & 3 & 100.0 \\
\hline Burseraceae & Bursera simaruba (L.) Sarg. & PA-25 & chaca & & leaves & fever & $\mathbf{P}$ & fresh & cataplasm & 45 & 100.0 \\
\hline Cactaceae & $\begin{array}{l}\text { Opuntia ficus-indica (L.) } \\
\text { Mill. }\end{array}$ & PA-26 & $\begin{array}{l}\text { Nopal, pepino } \\
\text { and cascara }\end{array}$ & $\mathrm{N}$ & leaves & cholesterol & B & fresh & liquefied & 2 & 66.7 \\
\hline & & & & & & $\begin{array}{l}\text { clean stomach } \\
\text { diabetes }\end{array}$ & $\begin{array}{l}\text { G } \\
\mathrm{H}\end{array}$ & $\begin{array}{l}\text { fresh } \\
\text { fresh }\end{array}$ & $\begin{array}{l}\text { liquefied } \\
\text { decocted }\end{array}$ & $\begin{array}{l}8 \\
2\end{array}$ & \\
\hline Cannabacea & Cannabis sativa $\mathrm{L}$. & PA-27 & marihuana & Ex & $\begin{array}{l}\text { whole } \\
\text { plant }\end{array}$ & rheumatism & E & fresh & tincture & 9 & 100.0 \\
\hline
\end{tabular}


Table 2. Cont.

\begin{tabular}{|c|c|c|c|c|c|c|c|c|c|c|c|}
\hline Family & Scientific Name & $\begin{array}{c}\text { Voucher } \\
\text { Specimen }\end{array}$ & $\begin{array}{l}\text { Common } \\
\text { Name }\end{array}$ & Origin & $\begin{array}{l}\text { Plant Part } \\
\text { Used }\end{array}$ & Ailments & Category & $\begin{array}{l}\text { Preparation } \\
\text { Mode }\end{array}$ & $\begin{array}{c}\text { Detailed } \\
\text { Administration }\end{array}$ & UR & FL (\%) \\
\hline \multirow[t]{2}{*}{ Cannabaceae } & \multirow[t]{2}{*}{ Trema micrantha (L) Blume } & \multirow[t]{2}{*}{ PA-28 } & \multirow{2}{*}{ puam } & \multirow[t]{2}{*}{$\mathrm{N}$} & \multirow[t]{2}{*}{ leaves } & chicken pox & $\mathbf{P}$ & fresh & bath & 8 & 100.0 \\
\hline & & & & & & measles & $\mathbf{P}$ & fresh & bath & 6 & \\
\hline Caricaceae & Carica papaya $L$. & PA-29 & papaya & & stem & pain in ears & $\mathrm{Q}$ & fresh & burned & 5 & 100.0 \\
\hline \multirow{2}{*}{ Chenopodiaceae } & \multirow[t]{2}{*}{ Chenopodium ambrosioides } & \multirow[t]{2}{*}{ PA-30 } & \multirow[t]{2}{*}{ epasote } & \multirow[t]{2}{*}{$\mathrm{N}$} & \multirow[t]{2}{*}{ leaves } & intestinal worms & $\mathrm{G}$ & fresh & infusion & 13 & 100.0 \\
\hline & & & & & & stomach ache & G & fresh & infusion & 12 & \\
\hline \multirow[t]{6}{*}{ Commelinaceae } & \multirow[t]{6}{*}{ Tradescantia spathacea Sw. } & \multirow[t]{6}{*}{ PA-31 } & \multirow[t]{6}{*}{$\begin{array}{c}\text { barquilla, } \\
\text { maguey } \\
\text { morado }\end{array}$} & \multirow[t]{6}{*}{$\mathrm{N}$} & \multirow[t]{6}{*}{ leaves } & grains in the mouth & $\mathrm{L}$ & fresh & squeezed & 3 & 38.7 \\
\hline & & & & & & kidney problem & $\mathrm{C}$ & fresh & infusion & 8 & \\
\hline & & & & & & respiratory system & A & fresh & infusion & 8 & \\
\hline & & & & & & wounds & $\mathrm{O}$ & fresh & burned & 1 & \\
\hline & & & & & & skin infection & $\mathrm{O}$ & fresh & bath & 3 & \\
\hline & & & & & & wounds and bruises & $\mathrm{O}$ & fresh & cataplasm & 8 & \\
\hline Cucurbitaceae & Cucurbita pepo L. & PA-32 & calabaza & $\mathrm{N}$ & latex & scratches, wounds & $\mathrm{O}$ & fresh & squeezed & 4 & 100.0 \\
\hline Cucurbitaceae & Sechium edule (Jacq.) Sw. & PA-33 & chayote & $\mathrm{N}$ & fruit & cholesterol & B & fresh & decocted & 12 & 100.0 \\
\hline Euphorbiaceae & $\begin{array}{l}\text { Cnidoscolus chayamansa Mc } \\
\text { Vaugh }\end{array}$ & PA-34 & chaya & $\mathrm{N}$ & leaves & high pressure & B & fresh & decocted & 7 & 100.0 \\
\hline \multirow[t]{3}{*}{ Euphorbiaceae } & \multirow{3}{*}{$\begin{array}{l}\text { Cnidoscolus tubulosus } \\
\text { (Müll.Arg.) I.M.Johnst. }\end{array}$} & \multirow[t]{3}{*}{ PA-35 } & \multirow[t]{3}{*}{$\begin{array}{l}\text { hortiga macho } \\
\text { con espina }\end{array}$} & \multirow[t]{3}{*}{$\mathrm{N}$} & root & kidney problem & C & dry & decocted & 5 & 61.1 \\
\hline & & & & & stem & kidney problem & C & fresh & infusion & 6 & \\
\hline & & & & & latex & tooth pain & $\mathrm{L}$ & fresh & raw & 7 & \\
\hline Euphorbiaceae & Euphorbia hirta L. & PA-36 & riñonina & $\mathrm{N}$ & leaves & kidney problem & C & fresh & infusion & 6 & 100.0 \\
\hline \multirow{3}{*}{ Euphorbiaceae } & \multirow[t]{3}{*}{ Jatropha curcas L. } & \multirow[t]{3}{*}{ PA-37 } & piñon & $\mathrm{N}$ & latex & bleeding of the gums, & L & fresh & topical raw & 4 & 57.1 \\
\hline & & & & & leaves & grains & $\mathrm{O}$ & fresh & bath & 1 & \\
\hline & & & & & latex & herpes & $\mathrm{P}$ & fresh & topical raw & 2 & \\
\hline Fabaceae & Bauhinia divaricata $\mathrm{L}$. & PA-38 & pata de vaca & $\mathrm{N}$ & leaves & diabetes & H & fresh & infusion & 4 & 44.4 \\
\hline & & & & & & diarrhoea & G & fresh & infusion & 3 & \\
\hline & & & $\begin{array}{l}\text { Mixed with } \\
\text { crushed rice }\end{array}$ & & $\begin{array}{l}\text { whole } \\
\text { plant }\end{array}$ & dysentery & G & fresh & decocted & 1 & \\
\hline & & & & & leaves & grains in the skin & $\mathrm{O}$ & fresh & bath & 1 & \\
\hline Fabaceae & Cassia fistula $\mathrm{L}$. & PA-39 & hojasen & Ex & leaves & colitis & G & fresh & infusion & 3 & 100.0 \\
\hline Fabaceae & $\begin{array}{c}\text { Erythrina caribaea Krukoff } \\
\text { \& Barneby }\end{array}$ & PA-40 & pichoco & $\mathrm{N}$ & bark & push delivering in parthum & D & fresh & decocted & 2 & 100.0 \\
\hline Fabaceae & $\begin{array}{l}\text { Eysenhardtia polystachya } \\
\text { (Ortega) Sarg }\end{array}$ & PA-41 & $\begin{array}{l}\text { tarai (palo } \\
\text { azul) }\end{array}$ & $\mathrm{N}$ & bark & kidney problem & C & dry & infusion & 5 & 100.0 \\
\hline Fabaceae & $\begin{array}{l}\text { Gliricidia sepium (Jacq.) } \\
\text { Walp }\end{array}$ & PA-42 & cocohuite & $\mathrm{N}$ & leaves & fever & $\mathrm{P}$ & fresh & tincture & 2 & 100.0 \\
\hline Fabaceae & $\begin{array}{l}\text { Leucaena leисосерhala } \\
\text { (Lam.) de Wit }\end{array}$ & PA-43 & guaje & $\mathrm{N}$ & fruit & intestinal worms & G & fresh & raw & 4 & 100.0 \\
\hline Geraniaceae & Pelargonium spp. & PA-44 & $\begin{array}{c}\text { malva con } \\
\text { hortiga }\end{array}$ & Ex & leaves & chicken pox & $\mathrm{P}$ & fresh & decocted & 1 & 50.0 \\
\hline & & & & & & grains & $\mathrm{O}$ & fresh & decocted & 1 & \\
\hline Lamiaceae & Mentha spicata L. & PA-45 & hierva buena & Ex & leaves & colic pain & $\mathrm{D}$ & fresh & infusion & 14 & 57.6 \\
\hline
\end{tabular}


Table 2. Cont.

\begin{tabular}{|c|c|c|c|c|c|c|c|c|c|c|c|}
\hline Family & Scientific Name & $\begin{array}{c}\text { Voucher } \\
\text { Specimen }\end{array}$ & $\begin{array}{l}\text { Common } \\
\text { Name }\end{array}$ & Origin & $\begin{array}{l}\text { Plant Part } \\
\text { Used }\end{array}$ & Ailments & Category & $\begin{array}{l}\text { Preparation } \\
\text { Mode }\end{array}$ & $\begin{array}{c}\text { Detailed } \\
\text { Administration }\end{array}$ & UR & FL (\%) \\
\hline \multirow{7}{*}{ Lamiaceae } & & & & & & stomach ache & G & fresh & decocted & 19 & \\
\hline & Осітит basilicum $\mathrm{L}$. & PA-46 & albacahar & $\mathrm{N}$ & leaves & anxiety & $\mathrm{F}$ & fresh & raw & 7 & 36.0 \\
\hline & & & & & & bad wind & $\mathrm{s}$ & fresh & raw & 3 & \\
\hline & & & & & & $\operatorname{dizzy}$ & $\mathrm{Q}$ & fresh & infusion & 9 & \\
\hline & & & & & & evil eye & $\mathrm{s}$ & fresh & bath & 4 & \\
\hline & & & & & & high pressure & B & fresh & infusion & 1 & \\
\hline & & & & & & nausea & G & fresh & infusion & 1 & \\
\hline Lamiaceae & Salvia spp. & PA- 47 & salvia & En & leaves & spasm & E & fresh & decocted & 2 & 100.0 \\
\hline \multirow[t]{5}{*}{ Lauraceae } & Сinnатотит verum J.Presl & PA-48 & $\begin{array}{l}\text { mango con } \\
\text { canela }\end{array}$ & Ex & leaves & abortive & $\mathbf{D}$ & fresh & decocted & 2 & 58.3 \\
\hline & & & & & & chinkunguya & $\mathrm{P}$ & fresh & decocted & 2 & \\
\hline & & & & & & cough & A & fresh & decocted & 1 & \\
\hline & & & & & & colic pain & D & fresh & decocted & 5 & \\
\hline & & & & & & drink & $\mathrm{s}$ & fresh & decocted & 2 & \\
\hline Lauraceae & Persea americana Mill. & PA-49 & aguacate & $\mathrm{N}$ & seed & abortive & D & fresh & decocted & 5 & 86.2 \\
\hline \multirow[t]{4}{*}{ Lauraceae } & Persea americana Mill. & & $\begin{array}{l}\text { aguacate } \\
\text { oloroso }\end{array}$ & $\mathrm{N}$ & leaves & diarrhoea & G & fresh & infusion & 19 & \\
\hline & & & & & seed & kidney problem & C & fresh & liquefied & 3 & \\
\hline & & & & & leaves & nausea & G & fresh & infusion & 2 & \\
\hline & & & & & leaves & stomach ache & G & fresh & infusion & 29 & \\
\hline \multirow[t]{3}{*}{ Loranthaceae } & $\begin{array}{l}\text { Struthanthus crassipes } \\
\text { (Oliver) Eichl. }\end{array}$ & PA-50 & secapalo & En & leaves & grains & o & fresh & decocted & 7 & 53.3 \\
\hline & & & & & & kidney problem & $\mathrm{C}$ & fresh & decocted & 7 & \\
\hline & & & & & & wounds & $\mathbf{O}$ & fresh & bath & 1 & \\
\hline \multirow[t]{6}{*}{ Malvaceae } & Guazum aulmifolia Lam. & PA-51 & guazima & $\mathrm{N}$ & bark & colitis & G & dry & decocted & 5 & 61.9 \\
\hline & & & & & bark & diabetes & $\mathrm{H}$ & dry & decocted & 4 & \\
\hline & & & & & bark & diarrhoea & G & dry & decocted & 4 & \\
\hline & & & & & fruit & drink & $\mathrm{s}$ & fresh & squeezed & 2 & \\
\hline & & & & & bark & stomach ache & G & dry & decocted & 4 & \\
\hline & & & & & leaves & veterinary & $\mathrm{R}$ & fresh & raw & 2 & \\
\hline Malvaceae & $\begin{array}{l}\text { Heliocarpus appendiculatus } \\
\text { Turcz. }\end{array}$ & PA-52 & jonote & $\mathrm{N}$ & latex & wounds & $\mathrm{O}$ & dry & topical raw & 4 & 100.0 \\
\hline \multirow[t]{2}{*}{ Malvaceae } & Sida rhombifolia $\mathrm{L}$. & PA-53 & $\begin{array}{l}\text { malva and } \\
\text { albacahar }\end{array}$ & $\mathrm{N}$ & leaves & bad wind & $\mathrm{s}$ & fresh & raw & 1 & 100.0 \\
\hline & & & & & stem & ritual & $\mathrm{S}$ & fresh & raw & 4 & \\
\hline Malvaceae & $\begin{array}{l}\text { Sphaeralcea angustifolia } \\
\text { (Cav.) G.Don }\end{array}$ & PA-54 & $\begin{array}{l}\text { hierva del } \\
\text { negro }\end{array}$ & $\mathrm{N}$ & $\begin{array}{l}\text { whole } \\
\text { plant }\end{array}$ & bad wind & s & fresh & raw & 15 & 100.0 \\
\hline Meliaceae & Azadirachta indica A. Juss. & PA-55 & neem & Ex & fruit & diabetes & $\mathbf{H}$ & fresh & infusion & 18 & 100.0 \\
\hline \multirow[t]{4}{*}{ Meliaceae } & Cedrela odorata $\mathrm{L}$. & PA-56 & cedro & $\mathrm{N}$ & bark & abortive & D & dry & decocted & 4 & 75.0 \\
\hline & & & & & bark & fever & $\mathrm{P}$ & dry & decocted & 1 & \\
\hline & & & & & leaves & inflammation & E & fresh & decocted & 1 & \\
\hline & & & & & bark & $\begin{array}{l}\text { problems in trying to have } \\
\text { children }\end{array}$ & D & dry & decocted & 2 & \\
\hline
\end{tabular}


Table 2. Cont.

\begin{tabular}{|c|c|c|c|c|c|c|c|c|c|c|c|}
\hline Family & Scientific Name & $\begin{array}{l}\text { Voucher } \\
\text { Specimen }\end{array}$ & $\begin{array}{l}\text { Common } \\
\text { Name }\end{array}$ & Origin & $\begin{array}{l}\text { Plant Part } \\
\text { Used }\end{array}$ & Ailments & Category & $\begin{array}{l}\text { Preparation } \\
\text { Mode }\end{array}$ & $\begin{array}{c}\text { Detailed } \\
\text { Administration }\end{array}$ & UR & FL (\%) \\
\hline Meliaceae & Melia azedarach L. & PA-57 & piocha & Ex & leaves & diabetes & $\mathrm{H}$ & fresh & infusion & 8 & 100.0 \\
\hline Monimiaceae & Peumus boldus Molina & PA-58 & boldo & Ex & leaves & colitis & G & fresh & infusion & 4 & 100.0 \\
\hline \multirow[t]{2}{*}{ Moraceae } & Morus celtidifolia Kunth & PA-59 & mora & $\mathrm{N}$ & leaves & chinkunguya & $\mathbf{P}$ & fresh & decocted & 2 & 66.7 \\
\hline & & & & & leaves & tooth pain & $\mathrm{L}$ & fresh & raw & 1 & \\
\hline \multirow[t]{2}{*}{ Moringaceae } & Moringa oleifera Lam. & PA-60 & moringa & Ex & leaves & cancer & I & fresh & decocted & 1 & 85.7 \\
\hline & & & & & leaves & diabetes & $\mathbf{H}$ & fresh & decocted & 6 & \\
\hline \multirow[t]{2}{*}{ Musaceae } & Musa spp. & PA-61 & platano & Ex & bark & respiratory system & A & fresh & decocted & 2 & 77.8 \\
\hline & & & & & bark & tuberculosis & $\mathbf{P}$ & fresh & fermented & 7 & \\
\hline \multirow[t]{2}{*}{ Myrsinaceae } & Ardisia compressa Kunth & PA-62 & $\begin{array}{l}\text { capulin and } \\
\text { nona }\end{array}$ & $\mathrm{N}$ & leaves & stomach ache & G & fresh & infusion & 5 & 62.5 \\
\hline & & & & & leaves & wounds & $\mathrm{O}$ & fresh & bath & 3 & \\
\hline Myrtaceae & Eucalyptus globulus Labill & PA-63 & eucalipto & Ex & leaves & bronchitis & A & fresh & decocted & 3 & 100.0 \\
\hline Myrtaceae & Pimenta dioica $(\mathrm{L}$.$) Merr.$ & PA-64 & pimienta & Ex & leaves & flu & A & fresh & decocted & 3 & 100.0 \\
\hline \multirow[t]{2}{*}{ Myrtaceae } & Psidium guajava $\mathrm{L}$. & PA-65 & guayaba & $\mathrm{N}$ & leaves & diarrhoea & G & dry & decocted & 16 & 53.3 \\
\hline & & & & & bark & flu & A & dry & decocted & 14 & \\
\hline Myrtaceae & $\begin{array}{l}\text { Syzygium aromaticum (L.) } \\
\text { Merr. \& Perry }\end{array}$ & PA-66 & clavo & Ex & seed & tooth pain & $\mathrm{L}$ & dry & topical raw & 5 & 100.0 \\
\hline Nyctaginaceae & Bougainville aglabra Choisy & PA-67 & bugambilia & Ex & flower & cough & A & fresh & infusion & 19 & 100.0 \\
\hline \multirow[t]{3}{*}{ Orchidaceae } & $\begin{array}{l}\text { Vanilla planifolia Jacks. ex } \\
\text { Andrews }\end{array}$ & PA-68 & vainilla & $\mathrm{N}$ & fruit & drink & $\mathrm{s}$ & fresh & fermented & 2 & 72.7 \\
\hline & & & & & fruit & drink & $\mathrm{s}$ & fresh & raw & 6 & \\
\hline & & & & & fruit & menopause & $\mathrm{D}$ & fresh & tincture & 1 & \\
\hline Papaveraceae & Fumaria officinalis L. & PA-69 & $\begin{array}{l}\text { Sangre de } \\
\text { Cristo (Blood } \\
\text { of Christ) }\end{array}$ & Ex & leaves & wounds & $\mathrm{O}$ & fresh & bath & 3 & 100.0 \\
\hline Passifloraceae & Passiflora coriacea Juss. & PA-70 & $\begin{array}{l}\text { hierva del } \\
\text { murcielago }\end{array}$ & $\mathrm{N}$ & leaves & kidney problem & C & fresh & decocted & 6 & 100.0 \\
\hline Pedaliaceae & Sesamum indicum L. & PA-71 & ajonjoli & Ex & seed & breastfeeding & $\mathrm{D}$ & dry & decocted & 15 & 100.0 \\
\hline \multirow[t]{6}{*}{ Piperaceae } & Peperomia granulosa Trel. & PA-72 & $\begin{array}{l}\text { gordonzillo } \\
\text { (acovo) }\end{array}$ & $\mathrm{N}$ & root & breastfeeding & D & fresh & decocted & 5 & 53.1 \\
\hline & & & & & stems & menstruation & D & fresh & decocted & 4 & \\
\hline & & & & & root & to have children & D & fresh & decocted & 8 & \\
\hline & & & & & leaves & cirrhosis & $\mathrm{N}$ & fresh & infusion & 1 & \\
\hline & & & & & leaves & rheumatism & E & fresh & burned & 9 & \\
\hline & & & $\begin{array}{c}\text { acoyo } \\
\text { (gordonsillo) } \\
\text { and ajo }\end{array}$ & & leaves & respiratory system & A & fresh & infusion & 5 & \\
\hline
\end{tabular}


Table 2. Cont.

\begin{tabular}{|c|c|c|c|c|c|c|c|c|c|c|c|}
\hline Family & Scientific Name & $\begin{array}{c}\text { Voucher } \\
\text { Specimen }\end{array}$ & $\begin{array}{l}\text { Common } \\
\text { Name }\end{array}$ & Origin & $\begin{array}{l}\text { Plant Part } \\
\text { Used }\end{array}$ & Ailments & Category & $\begin{array}{l}\text { Preparation } \\
\text { Mode }\end{array}$ & $\begin{array}{c}\text { Detailed } \\
\text { Administration } \\
\end{array}$ & UR & FL (\%) \\
\hline Piperaceae & $\begin{array}{l}\text { Piper sanctum (Miq.) } \\
\text { Schltdl. ex C.DC. }\end{array}$ & PA-73 & hierva santa & $\mathrm{N}$ & leaves & clean baby and post-parthum & $\mathrm{D}$ & fresh & decocted & 2 & 100.0 \\
\hline Plantaginaceae & $\begin{array}{c}\text { Plantago major } \mathrm{L} \text {. } \\
\text { Cymbopogon citratu }\end{array}$ & PA-74 & llanten & Ex & leaves & skin problems & $\mathrm{O}$ & fresh & decocted & 15 & 100.0 \\
\hline Poaceae & $\begin{array}{c}\text { Cymbopogon citratus (DC.) } \\
\text { Stapf }\end{array}$ & PA-75 & zacate limon & Ex-invader & leaves & drink & $\mathrm{s}$ & fresh & & 7 & 100.0 \\
\hline \multirow[t]{4}{*}{ Poaceae } & $\begin{array}{l}\text { Pachystachys spicata (Ruiz } \\
\text { \& Pav.) Wassh. }\end{array}$ & PA-76 & mohuite & $\mathrm{N}$ & stem & bad wind & $\mathrm{S}$ & fresh & & 1 & 70.6 \\
\hline & & & & & leaves & epilepsy & $\mathrm{F}$ & fresh & decocted & 3 & \\
\hline & & & & & & kidney problem & C & fresh & decocted & 12 & \\
\hline & & & & & & nausea & G & fresh & infusion & 1 & \\
\hline Poaceae & Phalaris canariensis $\mathrm{L}$. & PA-77 & alpistle & Ex & seed & diabetes & $\mathrm{H}$ & fresh & liquefied & 9 & 100.0 \\
\hline \multirow[t]{2}{*}{ Poaceae } & Zea maiz L. & PA-78 & maiz morado & $\mathrm{N}$ & seed & alcoholic drink & $\mathrm{s}$ & fresh & fermented & 3 & 82.4 \\
\hline & & & pelo de maiz & & fruit & kidney problem & C & fresh & infusion & 14 & \\
\hline Portulacaceae & Portulaca oleraceae L. & PA-79 & verdolaga & $\mathrm{N}$ & leaves & blood circulation & B & fresh & burned & 2 & 100.0 \\
\hline Rosaceae & $\begin{array}{l}\text { Eriobotrya japonica } \\
\text { (Thunb.) Lindl. }\end{array}$ & PA- 80 & nispero & Ex & leaves & kidney problem & C & fresh & decocted & 2 & 100.0 \\
\hline Rosaceae & Prunus domestica $\mathrm{L}$. & PA-81 & ciruela & Ex & leaves & $\begin{array}{l}\text { rash and grains } \\
\text { smallpox }\end{array}$ & $\begin{array}{l}\mathrm{O} \\
\mathrm{P}\end{array}$ & $\begin{array}{l}\text { fresh } \\
\text { fresh }\end{array}$ & $\begin{array}{l}\text { smashed } \\
\text { bath }\end{array}$ & $\begin{array}{l}4 \\
2\end{array}$ & 66.7 \\
\hline \multirow[t]{15}{*}{ Rubiaceae } & Hamelia patens Jacq. & PA-82 & tres hojitas & $\mathrm{N}$ & leaves & anaemia & B & fresh & infusion & 3 & 23.4 \\
\hline & & & & & & blood circulation & B & fresh & decocted & 7 & \\
\hline & & & & & & breastfeeding & $\mathrm{D}$ & fresh & burned & 6 & \\
\hline & & & & & & cancer & I & dry & decocted & 9 & \\
\hline & & & & & & colitis & G & fresh & decocted & 4 & \\
\hline & & & & & & diabetes & $\mathbf{H}$ & fresh & decocted & 7 & \\
\hline & & & & & & diabetes & $\mathbf{H}$ & fresh & infusion & 11 & \\
\hline & & & & & & gastritis & G & fresh & infusion & & \\
\hline & & & & & & gastritis & G & fresh & decocted & 8 & \\
\hline & & & & & & high pressure & B & fresh & infusion & 1 & \\
\hline & & & & & & menstruation & $\mathrm{D}$ & fresh & decocted & 2 & \\
\hline & & & & & root & respiratory system & A & dry & decocted & 7 & \\
\hline & & & & & & skin problems, fungus & $\mathrm{O}$ & fresh & squeezed & 2 & \\
\hline & & & & & & ulcers & G & fresh & decocted & 5 & \\
\hline & & & & & & wounds & $\mathrm{O}$ & fresh & bath & 5 & \\
\hline \multirow[t]{2}{*}{ Rubiaceae } & Morinda citrifolia $\mathrm{L}$. & PA-83 & noni & Ex & fruit & diabetes & $\mathbf{H}$ & fresh & liquefied & 10 & 83.3 \\
\hline & & & & & & heart problems & B & fresh & squeezed & 2 & \\
\hline \multirow[t]{5}{*}{ Rutaceae } & Casimiroa edulis La Llave & PA-84 & zapote blanco & $\mathrm{N}$ & leaves & cholesterol & B & fresh & infusion & 3 & 30.0 \\
\hline & & & & & latex & gum & $\mathrm{L}$ & dry & raw & 2 & \\
\hline & & & & & bark & diabetes & $\mathrm{H}$ & dry & decocted & 1 & \\
\hline & & & & & leaves & fever & $\mathrm{P}$ & fresh & infusion & 2 & \\
\hline & & & & & bark & kidney problem & C & dry & decocted & 2 & \\
\hline Rutaceae & $\begin{array}{l}\text { Citrus aurantiifolia } \\
\text { (Christm.) Swingle }\end{array}$ & PA-85 & $\begin{array}{l}\text { azares de } \\
\text { naranjo }\end{array}$ & Ex & leaves & anxiety & $\mathbf{F}$ & fresh & decocted & 3 & 100.0 \\
\hline
\end{tabular}


Table 2. Cont.

\begin{tabular}{|c|c|c|c|c|c|c|c|c|c|c|c|}
\hline Family & Scientific Name & $\begin{array}{c}\text { Voucher } \\
\text { Specimen }\end{array}$ & $\begin{array}{l}\text { Common } \\
\text { Name }\end{array}$ & Origin & $\begin{array}{l}\text { Plant Part } \\
\text { Used }\end{array}$ & Ailments & Category & $\begin{array}{l}\text { Preparation } \\
\text { Mode }\end{array}$ & $\begin{array}{c}\text { Detailed } \\
\text { Administration }\end{array}$ & UR & FL $(\%)$ \\
\hline \multirow[t]{3}{*}{ Rutaceae } & Citrus $\times$ aurantium $\mathrm{L}$. & PA-86 & naranja cucha & Ex & leaves & anxiety & F & fresh & decocted & 9 & 64.3 \\
\hline & & & & & & cough & A & fresh & decocted & 4 & \\
\hline & & & $\begin{array}{c}\text { naranja, } \\
\text { papaya, limon, } \\
\text { nopal }\end{array}$ & & fruit & diabetes & $\mathrm{H}$ & fresh & liquefied & 1 & \\
\hline \multirow[t]{2}{*}{ Rutaceae } & Citrus limetta Risso & PA- 87 & lima chichi & Ex & fruit & high pressure & B & fresh & decocted & 3 & 75.0 \\
\hline & & & & & & infection in the eyes & $\mathrm{Q}$ & fresh & squeezed & 9 & \\
\hline Rutaceae & Citrus medica $\mathrm{L}$. & PA-88 & limon & $\mathrm{N}$ & fruit & cough & $\widehat{A}$ & fresh & infusion & 14 & \\
\hline \multirow[t]{2}{*}{ Rutaceae } & Citrus sinensis (L.) Osbeck & PA-89 & naranja & Ex & leaves & anxiety & F & fresh & infusion & 6 & 54.5 \\
\hline & & & & & & flu & A & fresh & infusion & 5 & \\
\hline \multirow[t]{2}{*}{ Rutaceae } & $\begin{array}{l}\text { Murraya paniculata (L.) } \\
\text { Jack }\end{array}$ & PA-90 & limonaria & Ex & leaves & diabetes & $\mathbf{H}$ & fresh & squeezed & 2 & 66.7 \\
\hline & & & & & & tooth pain & $\mathrm{L}$ & fresh & decocted & 1 & \\
\hline \multirow[t]{8}{*}{ Rutaceae } & Ruta graveolens L. & PA-91 & ruda & Ex & leaves & colitis & G & fresh & infusion & 3 & 43.8 \\
\hline & & & & & & evil eye & $\mathrm{s}$ & fresh & raw & 3 & \\
\hline & & & & & & gastritis & G & fresh & infusion & 4 & \\
\hline & & & & & & high pressure & B & fresh & infusion & 4 & \\
\hline & & & & & & menstruation & D & fresh & infusion & 8 & \\
\hline & & & & & & pain in ears & $\mathrm{Q}$ & fresh & infusion & 3 & \\
\hline & & & & & & abortive & D & fresh & infusion & 6 & \\
\hline & & & & & & pain in the chest & A & fresh & infusion & 1 & \\
\hline \multirow[t]{3}{*}{ Sapotaceae } & $\begin{array}{l}\text { Manilkara chicle (Pittier) } \\
\text { Gilly }\end{array}$ & PA-92 & $\begin{array}{l}\text { zapote chico } \\
\text { and guia del } \\
\text { chavote }\end{array}$ & $\mathrm{N}$ & leaves & cholesterol & B & fresh & infusion & 4 & 57.1 \\
\hline & & & & & & diabetes & $\mathrm{H}$ & fresh & infusion & 1 & \\
\hline & & & & & & high pressure & B & fresh & infusion & 2 & \\
\hline \multirow[t]{3}{*}{ Sapotaceae } & $\begin{array}{l}\text { Pouteria sapota (Jacq.) H.E. } \\
\text { Moore \& Stearn. }\end{array}$ & PA-93 & $\begin{array}{l}\text { zapote } \\
\text { rebentador }\end{array}$ & $\mathrm{N}$ & bark & diabetes & H & dry & decocted & 4 & 57.1 \\
\hline & & & & & fruit & diarrhoea & G & fresh & squeezed & 2 & \\
\hline & & & & & leaves & nausea & G & fresh & decocted & 1 & \\
\hline \multirow[t]{3}{*}{ Smilacaceae } & $\begin{array}{l}\text { Smilax mollis Humb. \& } \\
\text { Bonpl. ex Willd. }\end{array}$ & PA-94 & $\begin{array}{l}\text { cocolmecate } \\
\text { (bejuco } \\
\text { enredadera) }\end{array}$ & $\mathrm{N}$ & root & diabetes & $\mathbf{H}$ & dry & decocted & 4 & 57.1 \\
\hline & & & & & root & gastritis & G & dry & decocted & 1 & \\
\hline & & & & & bark & loss weight & B & dry & decocted & 2 & \\
\hline Solanaceae & $\begin{array}{c}\text { Physalis ixocarpa Brot. ex } \\
\text { Hornem }\end{array}$ & PA-95 & tomate verde & $\mathrm{N}$ & leaves & kidney problem & C & fresh & infusion & 4 & 100.0 \\
\hline Urticaceae & Cecropia obtusifolia Bertol. & PA-96 & $\begin{array}{l}\text { hormiguillo } \\
\text { (nihuiya) }\end{array}$ & $\mathrm{N}$ & bark & diabetes & $\mathbf{H}$ & dry & decocted & 2 & 100.0 \\
\hline \multirow[t]{2}{*}{ Verbenaceae } & Lippia duartei Moldenke & PA-97 & hierva dulce & $\mathrm{N}$ & $\begin{array}{l}\text { whole } \\
\text { plant }\end{array}$ & diabetes & H & dry & decocted & 1 & 50.0 \\
\hline & & & & & leaves & diarrhoea & G & dry & decocted & 1 & \\
\hline Verbenaceae & Lippia graveolens Kunth & PA-98 & oregano & $\mathrm{N}$ & leaves & respiratory system & A & dry & infusion & 4 & 100.0 \\
\hline
\end{tabular}


Table 2. Cont.

\begin{tabular}{|c|c|c|c|c|c|c|c|c|c|c|c|}
\hline Family & Scientific Name & $\begin{array}{c}\text { Voucher } \\
\text { Specimen }\end{array}$ & $\begin{array}{l}\text { Common } \\
\text { Name }\end{array}$ & Origin & $\begin{array}{l}\text { Plant Part } \\
\text { Used }\end{array}$ & Ailments & Category & $\begin{array}{l}\text { Preparation } \\
\text { Mode }\end{array}$ & $\begin{array}{c}\text { Detailed } \\
\text { Administration }\end{array}$ & UR & FL (\%) \\
\hline \multirow[t]{7}{*}{ Xanthorrhoeaceae } & \multirow[t]{7}{*}{ Aloe vera (L.) Burm.f. } & \multirow[t]{7}{*}{ PA-99 } & \multirow[t]{7}{*}{ savila } & \multirow[t]{7}{*}{ Ex } & \multirow[t]{3}{*}{ leaves } & gastritis & G & fresh & raw & 7 & \multirow[t]{7}{*}{33.3} \\
\hline & & & & & & hair problems & $\mathrm{Q}$ & fresh & smashed & 6 & \\
\hline & & & & & & inflammation & $\mathrm{E}$ & fresh & topical raw & 3 & \\
\hline & & & & & \multirow[t]{4}{*}{$\begin{array}{l}\text { whole } \\
\text { plant }\end{array}$} & ulcers & G & fresh & infusion & 3 & \\
\hline & & & & & & wounds & o & fresh & topical raw & 11 & \\
\hline & & & & & & anaemia & B & fresh & infusion & 1 & \\
\hline & & & & & & chinkunguya & $\mathrm{P}$ & fresh & infusion & 2 & \\
\hline \multirow[t]{2}{*}{ Zingiberaceae } & \multirow[t]{2}{*}{ Costus spicatus (Jacq.) Sw. } & \multirow[t]{2}{*}{ PA-100 } & \multirow{9}{*}{$\begin{array}{c}\text { caña de jabali } \\
\text { caña de jabali } \\
\text { con raiz de } \\
\text { chiote } \\
\text { gengibre }\end{array}$} & \multirow[t]{2}{*}{$\mathrm{N}$} & stem & kidney problem & C & dry & infusion & 21 & \multirow[t]{2}{*}{95.5} \\
\hline & & & & & root & hepatitis & $\mathrm{N}$ & dry & decocted & 1 & \\
\hline \multirow[t]{7}{*}{ Zingiberaceae } & \multirow[t]{7}{*}{ Zingiber officinale Roscoe } & \multirow[t]{7}{*}{ PA-101 } & & \multirow[t]{7}{*}{ Ex } & \multirow[t]{7}{*}{ root } & anaemia & B & fresh & decocted & 1 & \multirow[t]{7}{*}{54.5} \\
\hline & & & & & & blood circulation & B & fresh & decocted & 2 & \\
\hline & & & & & & clean the blood & B & fresh & raw & 3 & \\
\hline & & & & & & colic pain & $\mathrm{D}$ & fresh & decocted & 1 & \\
\hline & & & & & & stomach ache & G & fresh & decocted & 1 & \\
\hline & & & & & & intestinal worm & G & fresh & decocted & 2 & \\
\hline & & & & & & inflammation & E & fresh & decocted & 1 & \\
\hline
\end{tabular}

A, refers to Respiratory system disorders; B to Blood cardiovascular disorders; C, Kidney disorders; D, Genio-urinary disorders and childcare; E, Skeleton-muscular system disorders, F Nervous system disorders; G, Gastro-intestinal disorders; H, Endocrinal disorders; I, Oncology; L, Dental care; M, Poisonous bites; N, Liver disorders; O, Skin disorders; P, Fever and infective diseases; Q, Ear, eye, hair disorders; R, Veterinary uses, S, Different uses. N, native, En, endemic, Ex, exotic; Bold ailments treated, Main use of Plant on which FL\% is based. 


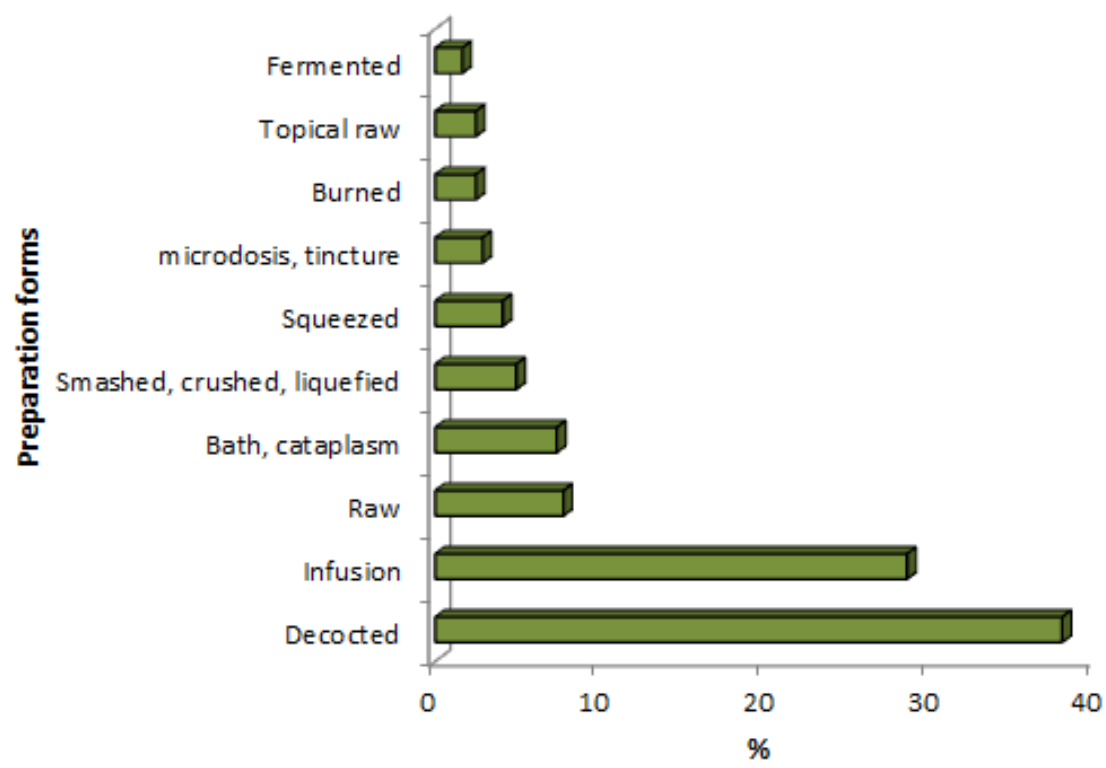

Figure 2. Remedies preparations expressed as percentages (\%).

\subsection{Use Reports, Informant's Consensus Factor and Fidelity Level}

Given the results of our observations, the studied communities had a significant variety of traditional uses, with a specific frame of ailments. A total of 77 ailments were grouped into 17 use-categories (Table 3) based upon the information gathered from those interviewed.

Table 3. Number and percent of use reports (UR) and important consensus factors (ICF) of the Mexican plant species for each ailment category.

\begin{tabular}{cccccc}
\hline $\mathbf{N}^{\circ}$ & AILMENT CATEGORIES & $\mathbf{N}^{\circ}$ SPECIES & $\mathbf{N}^{\circ}$ of UR & \% UR & ICF \\
\hline $\mathrm{A}$ & Respiratory system disorders & 17 & 100 & 7.89 & 0.84 \\
$\mathrm{~B}$ & Blood-cardiovascular disorders & 21 & 112 & 8.83 & 0.82 \\
$\mathrm{C}$ & Kidney disorders & 17 & 119 & 9.38 & 0.86 \\
$\mathrm{D}$ & Genital-urinary disorders and childcare & 17 & 118 & 9.31 & 0.86 \\
$\mathrm{E}$ & Skeleton-muscular system disorders & 8 & 34 & 2.68 & 0.79 \\
$\mathrm{~F}$ & Nervous system disorders & 5 & 28 & 2.21 & 0.85 \\
$\mathrm{G}$ & Gastro-intestinal disorders & 29 & 247 & 19.48 & 0.89 \\
$\mathrm{H}$ & Endocrinal disorders & 23 & 137 & 10.80 & 0.84 \\
$\mathrm{I}$ & Oncology & 5 & 44 & 3.47 & 0.91 \\
$\mathrm{~L}$ & Dental care & 9 & 27 & 2.13 & 0.69 \\
$\mathrm{M}$ & Poisonous bites & 2 & 14 & 1.10 & 0.92 \\
$\mathrm{~N}$ & Liver disorders & 3 & 3 & 0.24 & 0.00 \\
$\mathrm{O}$ & Skin disorders & 17 & 83 & 6.55 & 0.80 \\
$\mathrm{P}$ & Fever and infective diseases & 7 & 93 & 7.33 & 0.87 \\
$\mathrm{Q}$ & Ear-eye-hair disorders & 4 & 39 & 3.08 & 0.84 \\
$\mathrm{R}$ & Veterinary uses & 11 & 14 & 1.10 & 0.77 \\
$\mathrm{~S}$ & Different uses & 56 & 4.42 & 0.82 \\
\hline
\end{tabular}

UR, Use-reports; ICF, Informant's consensus factor.

The ICF was calculated for each ailment category, and the highest value was calculated for poisonous animal bites (ICF $=0.92$ ), which was for the roots of two plant species, Pentalinon andrieuxii (Müll.Arg.) B. F. Hansen \& Wunderlin (13 UR) and Allium sativum L. (1 UR), which were reported by informants to be used in tinctures for the treatment of snakebites. These species are the same species used to treat snakebite in Central America [27].

Problems related with different types of cancer (oncology use-category) showed an ICF of 0.91 with 5 species and 44 URs, followed by gastro-intestinal disorders (ICF $=0.89$ ), with 29 species and 247 
URs. Infective diseases and fever had an ICF of 0.87 with 13 species and 93 URs; kidney disorders and genital-urinary disorders reported a similar ICF, with 17 species associated with each. The use-category of liver disorders showed the lowest degree of consensus; only three informants mentioned three plant species to treat ailments belonging to this category (cirrhosis, hepatitis and liver disorders); probably informants had not exchanged their information. Malnutrition, poverty and environmental conditions are the main factors causing common ailments (digestive, respiratory and skin disorders), as previously reported $[28,29]$. This study also found that cancer (in the stomach, skin and gallbladder) and diabetes cases have increased recently, and this problem could be related to the diet in the region. Decoctions and infusions of leaves from Asclepias curassavica (16 URs), Rauvolfia tetraphylla (11 URs) and Hamelia patens (9 UR) were used to treat cancer, whereas plants, such as Tecoma stans, Psidium guajava, Hamelia patens, Persea americana and Anacardium occidentale, were included within the treatments for diabetes [30]. Matricaria recutita, Mentha spicata, Psidium guajava, and Chenopodium ambrosioides, are consumed in nine other Mexican states [5,31,32]. Aloe vera, Piper auritum, Rutha chalepensis, Citrus limon, Annona reticulate, and Cocos nucifera have been recorded to be widely used by indigenous people in central-southern Mexico [28,33].

The most commonly used species was Hamelia patens Jacq, with 77 URs. It is a large perennial shrub that has been used against a range of ailments by other indigenous communities in Mexico [34]. Totonacs use Hamelia patens to treat problems related to diabetes (UR = 18), gastrointestinal disorders (gastritis, colitis, and ulcers) (UR = 17), cancer (UR $=9)$, high blood pressure and blood circulation, respiratory problems, anaemia, breastfeeding, menstruation, skin disorders and wounds. Its medical effects has been proved in another countries, including India [35], in treatments of nervous shock for its antidepressant properties, athletes' foot, skin lesions, insect bites, inflammation, rheumatism, headache, asthma and dysentery.

The leaves of Persea americana are commonly used by the Mexicans in infusions to treat gastrointestinal problems; nevertheless, previous reports have shown an increased use of the bark and seeds against diabetes, cholesterol and kidney problems in Central America [36,37]. Currently, Aloe vera plays an important role for its pharmacological effectiveness in treating a large number of ailments, such as skin problems, gastrointestinal problems, blood circulation problems, kidney problems and malnutrition, but it has been widely used by people with diabetes in Central America [30]. The use of Aloe vera has been spread throughout Latin America; and its ease of management and reproduction provided a cheap option for industry and people to grow it.

Additionally, the prevalence of new health problems that are present in tropical areas, such as dengue and Chikungunya, is getting more common around the world, and the way to combat them is a challenge for the people. The local people use coconut water as a strategy to reduce the impact of dehydration with the effects caused by Chikungunya. They also boil the mango leaves and mix them with the coconut water to drink and manage the fever caused by mosquito-borne diseases.

Our findings are in line with Alonso-Castro, et al. [29], where the main reasons of why people use medicinal plants in Mexico are related to their effectiveness and the low cost of usage and acquisition. However, they use traditional medicine as a complementary alternative to modern medicine, which is becoming more commonly used to treat diseases in Mexico in the past few decades [38].

The present study shows FL values varying from $23.4 \%$ to $100 \%$ (Table 2). The results reported 50 medicinal plant species having maximum $100 \%$ FL. The high FL shows the preference of these plant species by informants for the treatment of specific diseases [39].

\section{Materials and Methods}

\subsection{Description of the Study Area}

The study area, geographically known as Totonacapan, is located in the northern part of Veracruz (Figure 3). It is a part of the Northern Gulf Coastal Plain with an area of $4300 \mathrm{~km}^{2}$. This region represents about $5.97 \%$ of the total area of the state of Veracruz, and comprises 15 municipalities. 
The study area belongs to the municipality of Papantla, known as Papanteca, with coordinates $20^{\circ} 27^{\prime} 39^{\prime \prime}$ $\mathrm{S}$ and $97^{\circ} 19^{\prime} 39^{\prime \prime}, \mathrm{W}$, and it lies at $180 \mathrm{~m}$ above sea level. It has an area of $1458.50 \mathrm{~km}^{2}$, which represents $2.03 \%$ of the state.

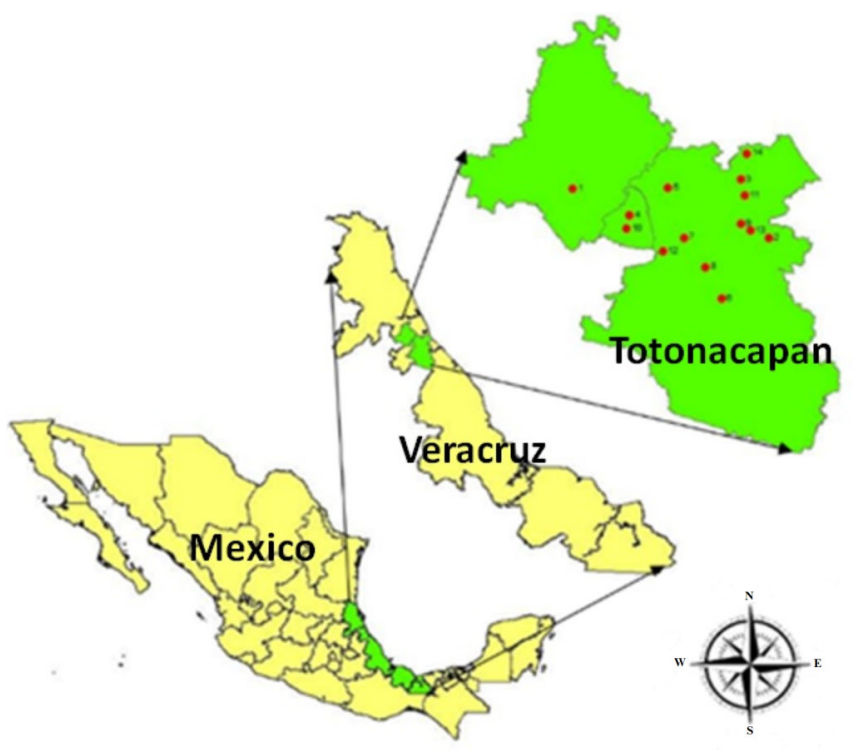

Figure 3. Study area of Veracruz, Mexico.

The climate is humid-warm, with an average annual temperature between 22 and $26{ }^{\circ} \mathrm{C}$. The annual rainfall in the area varies between 1000 and $1500 \mathrm{~mm}$. Totonacapan has a humid warm vegetation in most of its areas. The most common soils are Phaeozem-type Regosols and Vertisols, which are susceptible to erosion.

\subsection{Socio-Economic Description}

The municipal territory is mainly devoted to agriculture (68\%), followed by $11 \%$ livestock, $13 \%$ housing; the remaining $8 \%$ are occupied by trade, public offices and public spaces. The total population in the Totonacapan amounts to 622,846 inhabitants, of which 204,934 people form the economically active population (32.9\%). Nevertheless, it should be noted that $32.5 \%$ of Totonac's population work in the primary sector (agriculture, fisheries and forestry), and around 66,000 people in Papantla have indigenous roots. According to Rivera and Ruiz-Ramírez [40], 77 of the 212 municipalities in the state have moderate poverty.

The ethnic composition of Veracruz is quite diverse and complex. Veracruz is the third-highest state in terms of the number of indigenous people $(1,037,424)$ in México (CDI: National Commission for the Development of Indigenous Peoples) [41]. The state is divided into seven ethnic regions, and ethnic linguistic groups are located in the state of Veracruz. These groups still speak 14 different languages [Huasteco (Tenek), Popoluca, Mixe, Zoque, Chinanteco, Zapoteco, Mazateco, Mixteco, Otomí, Totonaca, Tepehua, Náhuatl from the Huasteca, Náhuatl from the Sierra de Zongolica, and Nahua from the south]. The most representative groups are Mazatecos, Totonacos and Zapotecos [40].

The Totonacos group lives in the city of Papantla and the surrounding areas; the tourism in this area helps them to sell handcrafts. Moreover, the performances of the sky dancers (Papantla flyers), and shamans are the main reason for tourism throughout the Mexican territory. It also has a remarkably high number of tourists who travel from different parts of the country to visit the shamans and buy the medicinal plants. The agriculture, livestock, forestry, traditional textile clothes, plants, fruits, practicing of traditional medicine and informal employment are the base of the local economy. Corn, beans, coffee, vanilla, bananas, citrons and oranges are the main crops produced in the region $[7,18,42]$. 
Previous studies performed in neighbour municipalities [18] have shown the number of farmers depending on the farm products, and discuss migration and a lack of good opportunities causing a loss of interest to preserve the traditional knowledge in the new generation. The study area is highly interesting, and represents a special combination of different factors to study the patterns of traditional medicine.

\subsection{Ethnobotanical Analysis}

Fieldwork was carried out from March to August of 2017 in 16 communities in the Papantla region (Adolfo López, Arroyo Grande, Carrizal, Cedros, Lahuas, Natividad, Panti, Papantla, Polutla, Poza Rica, Pozo Verde, San Antonio Xital, Veracruz, Spoupat, Volador and Zapotal). Before starting the survey, ethical approval for the study was first obtained from the indigenous organised group "Consejo de Ancianos de la Sabiduria Ancestral". Likewise, the president of the indigenous group, Miss María Luisa Santes Santes, supported and accompanied the visits to the people interviewed; this was in order to explain to them the investigation's purpose and to ask for their participation in this study. The questionnaires were supplied in the Spanish language and translated into the local language (Totonaco dialect) for the indigenous people who did not speak Spanish. Two translators accompanied the interviewer. Ethnobotanical information was collected from local inhabitants by using semi-structured questionnaires. A total of 85 informants were selected by snowball sampling. The informants were interviewed in their houses, in the streets and in local markets. Participant observation was also part of the interview, in order to have a better interpretation and analysis of the data reported by these informants.

The informants were asked to provide knowledge about the plant uses (local names, indication of use, used plant parts, places/methods/rituals of harvesting and administration mode). The informants were asked to show the place where they usually collect the plants. Many visits were conducted with the interviewed informants in order to collect, press and identify the medicinal plants. The plant material was collected by the authors and taxonomically identified. The botanical names of the species were verified with The Plant List (2013) (http://www.theplantlist.org) and voucher specimens were deposited in the Herbarium at the Chapingo Autonomous University.

\subsection{Data Analysis}

\subsubsection{Use Categories}

Based on the information obtained from the indigenous people in the study area, all of the reported ailments were categorised into use-categories. The basic structure of ethnobotanical information is the use-report (UR) and this can be considered as an individual report of a specific taxon/drug for a certain use-category. When a plant is cited as "used", it is considered as one "use-report", but if one informant mentions the same plant to treat more diseases in the same category, it is considered as a single use-report.

\subsubsection{Informants' Consensus Factor (ICF)}

In this study, the level of homogeneity among the information collected from diverse informants for plant species in treating particular diseases was calculated by the informants' consensus factor (ICF). It was estimated using the following formula [43]:

$$
I C F=\frac{N u r-N t}{N u r-1}
$$

where, Nur is the number of UR in each disease category and $N t$ is number of species used. Low ICF values suggest that the plant is used randomly, or information on its use is not exchanged among informants, whereas a high exchange of information and a well-defined selection criterion in the community is reported for a plant with high ICF values. 


\subsubsection{Fidelity level (FL)}

Fidelity level (FL) represents the percentage of informants claiming the use of a certain plant for the same major purpose, which can be calculated for the most frequently reported diseases or ailments as:

$$
\mathrm{FL}(\%)=(\mathrm{Np} / \mathrm{N}) \times 100
$$

where "Np" is the number of informants that claim a use of a plant species to treat a particular ailment, and " $\mathrm{N}$ " is the number of informants that use the plants as a medicine to treat any given disease or category [44].

\section{Conclusions}

The collected information indicates that the study area is rich in medicinal plants, and the results contribute to spread their uses. The social importance of the medicinal plants in the community is quite important for the public health and the conservation of traditional knowledge, and good management is required. In Papantla (Veracruz, Mexico), the indigenous population still depends upon medicinal plants to treat several ailments. The plants used have a mostly native origin. The species most frequently mentioned by the informants was Hamelia patens followed by Persea americana, Bursera simaruba, Matricaria chamomilla, Mentha spicata and Aloe vera. The safety and efficacy of cited plants needs to be investigated by phytochemical and pharmacological analysis, as it has been previously performed on several other traditionally used plant species [45-47].

Author Contributions: Conceptualization, E.F.C., J.M.Z.d.V and L.M.; Methodology, E.A.L.R., E.F.C., D.J.L.R., P.C., J.M.Z.d.V., L.M. and D.R.; Software, E.A.L.R., D.J.L.R., J.M.Z.d.V., E.F.C.; Validation, P.C., D.R. and L.M.; Formal Analysis, E.A.L.R., D.J.L.R., P.C., J.M.Z.d.V.; Investigation, E.F.C., L.M. and D.R.; Resources, E.F.C. and L.M.; Data Curation, E.A.L.R., E.F.C., D.J.L.R., P.C., J.M.Z.d.V., L.M. and D.R.; Writing - Original Draft Preparation, E.A.L.R., E.F.C., D.J.L.R., P.C., J.M.Z.d.V.; Writing - Review \& Editing, E.F.C. and L.M.; Supervision, E.F.C., L.M. and D.R.; Project Administration, L.M. and D.R.; Funding Acquisition, E.F.C. and L.M.

Funding: This work was supported by the Regione Basilicata; the Fondazione Enrico Mattei DGR n. 1490 del 4/12/2014, vs rep. n. 163 n8; and the Regional Project ALIMINTEGRA, GO NUTRIBAS financed on 16.1 PSR Basilicata founding ex D.G.R. n³12/17 CUP: C31G18000210002.

Acknowledgments: We would like to thank and acknowledge the local inhabitants of Papantla, Veracruz, Mexico for sharing their traditional medicinal plant knowledge.

Conflicts of Interest: The authors have no conflict of interest to declare.

\section{References}

1. Fidelis, Q.C.; Faraone, I.; Russo, D.; Aragão Catunda, F.E., Jr.; Vignola, L.; de Carvalho, M.G.; de Tommasi, N.; Milella, L. Chemical and Biological insights of Ouratea hexasperma (A. St.-Hil.) Baill: A source of bioactive compounds with multifunctional properties. Nat. Prod. Res. 2018, 33, 1500-1503. [CrossRef] [PubMed]

2. Mezrag, A.; Malafronte, N.; Bouheroum, M.; Travaglino, C.; Russo, D.; Milella, L.; Severino, L.; De Tommasi, N.; Braca, A.; Dal Piaz, F. Phytochemical and antioxidant activity studies on Ononis angustissima L. aerial parts: Isolation of two new flavonoids. Nat. Prod. Res. 2017, 31, 507-514. [CrossRef] [PubMed]

3. Chakraborty, T.; Saha, S.; Bisht, N. First report on the ethnopharmacological uses of medicinal plants by Monpa tribe from the Zemithang Region of Arunachal Pradesh, Eastern Himalayas, India. Plants 2017, 6, 13. [CrossRef] [PubMed]

4. Yabesh, J.E.M.; Prabhu, S.; Vijayakumar, S. An ethnobotanical study of medicinal plants used by traditional healers in silent valley of Kerala, India. J. Ethnopharmacol. 2014, 154, 774-789. [CrossRef] [PubMed]

5. Pérez-Nicolás, M.; Vibrans, H.; Romero-Manzanares, A.; Saynes-Vásquez, A.; Luna-Cavazos, M.; Flores-Cruz, M.; Lira-Saade, R. Patterns of knowledge and use of medicinal plants in Santiago Camotlán, Oaxaca, Mexico. Econc. Bot. 2017, 71, 209-223. [CrossRef]

6. Benabdesslem, Y.; Hachem, K.; Kahloula, K.; Slimani, M. Ethnobotanical Survey, Preliminary physico-chemical and phytochemical screening of Salvia argentea (L.) used by herbalists of the Saïda Province in Algeria. Plants 2017, 6, 59. [CrossRef] [PubMed] 
7. Srithi, K.; Balslev, H.; Wangpakapattanawong, P.; Srisanga, P.; Trisonthi, C. Medicinal plant knowledge and its erosion among the Mien (Yao) in northern Thailand. J. Ethnopharmacol. 2009, 123, 335-342. [CrossRef] [PubMed]

8. Leonti, M.; Vibrans, H.; Sticher, O.; Heinrich, M. Ethnopharmacology of the Popoluca, Mexico: An evaluation. J. Pharm. Pharmacol. 2001, 53, 1653-1669. [CrossRef] [PubMed]

9. Bye, R.; Linares, E.; Estrada, E. Biological diversity of medicinal plants in Mexico. In Phytochemistry of Medicinal Plants; Springer: Amsterdam, The Netherlands, 1995; pp. 65-82.

10. Coneval. Consejo nacional de evaluación de la politica de desarrollo social. In La Pobreza en la Población Indígena de México, 1st ed.; Julio: Coneval, Mexico, 2012; Volume 1, pp. 1-157. ISBN 978-607-9384-9301-9382.

11. Hernandez, T.; Canales, M.; Avila, J.G.; Duran, A.; Caballero, J.; de Vivar, A.R.; Lira, R. Ethnobotany and antibacterial activity of some plants used in traditional medicine of Zapotitlan de las Salinas, Puebla (Mexico). J Ethnopharmacol. 2003, 88, 181-188. [CrossRef]

12. Rzedowski, J. The Vegetation of Mexico; Editorial Limusa: México, Mexico, 1981.

13. Gheno-Heredia, Y.A.; Naba-Bernal, G.; Martínez-Campos, A.R.; SánchezVera, E. Las plantas medicinales de la organización de parteras y médicos indígenas tradicionales de Ixhuatlancillo, Veracruz, Mexico, y su significancia cultural. Polibotanica 2011, 31, 199-251.

14. Cano, L.; Torres, M.V.; Castillo, E.J. Flora Medicinal De Veracruz: Inventario Etnobotánico. 1; Universidad Veracruzana: Xalapa, Mexico, 1997.

15. Leonti, M. Traditional medicines and globalization: Current and future perspectives in ethnopharmacology. Front. Pharmacol. 2013, 4, 92. [CrossRef]

16. Dominguez-Barradas, C.; Eliseo Cruz-Morales, G.; Gonzalez-Gandara, C. Medicinal plants of the Ecological Reserve "Sierra of Otontepec" Township Chontla, Veracruz, Mexico. Cienciauat 2015, 9, 56-67. [CrossRef]

17. Cussy-Poma, V.; Fernández, E.; Rondevaldova, J.; Foffová, H.; Russo, D. Ethnobotanical inventory of medicinal plants used in the Qampaya District, Bolivia. B. Latinoam. Caribe Pl. 2017, 16, 68-77.

18. Burgos-Hernández, M.; Castillo-Campos, G.; Vergara Tenorio, M.D.C. Potentially useful flora from the tropical rainforest in central Veracruz, México: Considerations for their conservation. Acta. Bot. Mex. 2014, 109, 55-77. [CrossRef]

19. Sher, H.; Aldosari, A.; Ali, A.; de Boer, H.J. Indigenous knowledge of folk medicines among tribal minorities in Khyber Pakhtunkhwa, northwestern Pakistan. J. Ethnopharmacol. 2015, 166, 157-167. [CrossRef] [PubMed]

20. Nunkoo, D.H.; Mahomoodally, M.F. Ethnopharmacological survey of native remedies commonly used against infectious diseases in the tropical island of Mauritius. Ethnopharmacology 2012, 143, 548-564. [CrossRef] [PubMed]

21. Muller-Schwarze, N.K. Antes and hoy dia: Plant knowledge and categorization as adaptations to life in Panama in the twenty-first century. Econ. Bot. 2006, 60, 321-334. [CrossRef]

22. Case, R.J.; Pauli, G.F.; Soejarto, D.D. Factors in maintaining indigenous knowledge among ethnic communities of Manus Island. Econ. Bot. 2005, 59, 356-365. [CrossRef]

23. Ceuterick, M.; Vandebroek, I.; Torry, B.; Pieroni, A. Cross-cultural adaptation in urban ethnobotany: The Colombian folk pharmacopoeia in London. J. Ethnopharmacol. 2008, 120, 342-359. [CrossRef] [PubMed]

24. Leonti, M.; Ramirez, R.F.; Sticher, O.; Heinrich, M. Medicinal flora of the Popoluca, Mexico: A botanical systematical perspective. Econ. Bot. 2003, 57, 218-230. [CrossRef]

25. Mshana, G.; Hampshire, K.; Panter-Brick, C.; Walker, R. Urban-rural contrasts in explanatory models and treatment-seeking behaviours for stroke in Tanzania. J. Bios. Sci. 2008, 40, 35-52. [CrossRef] [PubMed]

26. Teklehaymanot, T.; Giday, M.; Medhin, G.; Mekonnen, Y. Knowledge and use of medicinal plants by people around Debre Libanos monastery in Ethiopia. J. Ethnopharmacol. 2007, 111, 271-283. [CrossRef] [PubMed]

27. Giovannini, P.; Howes, M.J.R.; Edwards, S.E. Data on medicinal plants used in Central America to manage diabetes and its sequelae (skin conditions, cardiovascular disease, kidney disease, urinary problems and vision loss). Data Brief 2016, 7, 1217-1220. [CrossRef] [PubMed]

28. Frei, B.; Baltisberger, M.; Sticher, O.; Heinrich, M. Medical ethnobotany of the Zapotecs of the Isthmus-Sierra (Oaxaca, Mexico): Documentation and assessment of indigenous uses. J. Ethnopharmacol. 1998, 62, 149-165. [CrossRef] 
29. Alonso-Castro, A.J.; Domínguez, F.; Maldonado-Miranda,J.J.; Castillo-Pérez, L.J.; Carranza-Álvarez, C.; Solano, E.; Isiordia-Espinoza, M.A.; del Carmen Juárez-Vázquez, M.; Zapata-Morales, J.R.; Argueta-Fuertes, M.A. Use of medicinal plants by health professionals in Mexico. J. Ethnopharmacol. 2017, 198, 81-86. [CrossRef]

30. Giovannini, P.; Howes, M.-J.R.; Edwards, S.E. Medicinal plants used in the traditional management of diabetes and its sequelae in Central America: A review. J. Ethnopharmacol. 2016, 184, 58-71. [CrossRef] [PubMed]

31. Amsterdam, J.D.; Li, Y.; Soeller, I.; Rockwell, K.; Mao, J.J.; Shults, J. A randomized, double-blind, placebo-controlled trial of oral Matricaria recutita (chamomile) extract therapy of generalized anxiety disorder. J. Clin. Psychopharm. 2009, 29, 378. [CrossRef] [PubMed]

32. Arqueta-Villamar, A.; Cano-Asseleih, L.; Rodarte, M. Atlas de las Plantas de la Medicina Tradicional Mexicana; Instituto Nacional Indigenista: Ciudad, Mexico, 1994; p. 348.

33. López, Z.; Núñez-Jinez, G.; Avalos-Navarro, G.; Rivera, G.; Salazar-Flores, J.; Ramírez, J.A.; Ayil-Gutiérrez, B.A.; Knauth, P. Antioxidant and cytotoxicological effects of Aloe vera food supplements. J. Food Qual. 2017. [CrossRef]

34. Salvador Gomez-Rivera, A.; Yuridia Lopez, C.; Martinez Burgos, M.; Cachon Mis, X.J. Morphological characterization of isolated endophyte fungi from Hamelia patens Jacq. y Lantana camara L. in Chetumal, Quintana Roo, Mexico. Teor. Y Prax. 2016, 12, 33-44. [CrossRef]

35. Surana, A.R.; Wagh, D.R.. GC-MS profiling and antidepressant-like effect of the extracts of Hamelia patens in animal model. Bangladesh J. Pharmacol. 2017, 12, 410-416. [CrossRef]

36. Imafidon, K.; Amaechina, F. Effects of aqueous seed extract of Persea americana Mill.(avocado) on blood pressure and lipid profile in hypertensive rats. Adv. Biol. Res. 2010, 4, 116-121.

37. Balick, M.J.; Arvigo, R.; Romero, L. The development of an ethnobiomedical forest reserve in Belize: Its role in the preservation of biological and cultural-diversity. Conserv. Biol. 1994, 8, 316-317. [CrossRef]

38. Robles-Zepeda, R.E.; Valenzuela-Antelo, O.; Garibay-Escobar, A.; Velázquez-Contreras, C.; Navarro-Navarro, M.; Contreras, L.R.; Corral, O.L.; Lozano-Taylor, J. Use of complementary and alternative medicine in a region of northwest Mexico. J. Altern. Complement. Med. 2011, 17, 787-788. [CrossRef] [PubMed]

39. Umair, M.; Altaf, M.; Abbasi, A.M. An ethnobotanical survey of indigenous medicinal plants in Hafizabad district, Punjab-Pakistan. PLoS ONE 2017, 12, e0177912. [CrossRef] [PubMed]

40. Rivera, A.F.; Ruiz-Ramírez, J. Grado de marginación en comunidades indígenas en Veracruz, México: Una percepción errónea de pobreza. Contrib. Cienc. Soc. 2014, 2014, 2.

41. INEGI. México en cifras. In Información Nacional Por Entidad Federativa y Municipios; Instituto Nacional de Estadística y Geografía de México (INEGI): Aguascalientes, Mexico, 2010.

42. de México, E.D.L.M. Instituto Nacional para el Federalismo y el Desarrollo Municipal. Gobierno del Estado de México, State Government of Mexico (Mexico). 2005. Available online: www.e-local.gob.mx/ (accessed on 25 February 2008).

43. Ferreira, F.S.; Brito, S.V.; Ribeiro, S.C.; Saraiva, A.A.; Almeida, W.O.; Alves, R.R. Animal-based folk remedies sold in public markets in Crato and Juazeiro do Norte, Ceará, Brazil. BMC Complementary Altern. Med. 2009, 9, 17. [CrossRef] [PubMed]

44. Appiah, K.; Oppong, C.; Mardani, H.; Omari, R.; Kpabitey, S.; Amoatey, C.; Onwona-Agyeman, S.; Oikawa, Y.; Katsura, K.; Fujii, Y. Medicinal Plants Used in the Ejisu-Juaben Municipality, Southern Ghana: An Ethnobotanical Study. Medicines 2019, 6, 1. [CrossRef] [PubMed]

45. 45 Gerbino, A.; Schena, G.; Milano, S.; Milella, L.; Franco Barbosa, A.; Armentano, F.; Procino, G.; Svelto, M.; Carmosino, M. Spilanthol from Acmella oleracea lowers the intracellular levels of cAMP impairing NKCC2 phosphorylation and water channel AQP2 membrane expression in mouse kidney. PLoS ONE 2016, 11, e0156021. [CrossRef] 
46. Russo, D.; Malafronte, N.; Frescura, D.; Imbrenda, G.; Faraone, I.; Milella, L.; Fernandez, E.; De Tommasi, N. Antioxidant activities and quali-quantitative analysis of different Smallanthus sonchifolius [(Poepp. and Endl.) H. Robinson] landrace extracts. Nat. Prod. Res. 2015, 29, 1673-1677. [CrossRef] [PubMed]

47. Ovesná, J.; Kučera, L.; Horníčková, J.; Svobodová, L.; Stavělíková, H.; Velíšek, J.; Milella, L. Diversity of Salk(en)yl cysteine sulphoxide content within a collection of garlic (Allium sativum L.) and its association with the morphological and genetic background assessed by AFLP. Sci. Hort. 2011, 129, 541-547. [CrossRef]

(C) 2019 by the authors. Licensee MDPI, Basel, Switzerland. This article is an open access article distributed under the terms and conditions of the Creative Commons Attribution (CC BY) license (http://creativecommons.org/licenses/by/4.0/). 